\title{
Nonlinear Hashin-Shtrikman bounds for hereditary problems
}

\author{
P. P. Procházka \& M. Toman \\ Czech Technical University in Prague, Civil Engineering, \\ Structural Mechanics, Czech Republic
}

\begin{abstract}
Classical Hashin-Shtrikman variational bounds are based on the assumption that all phases behave in a purely elastic and locally homogeneous manner. Hashin-Shtrikman principles, from which the bounds are derived, are extended in a paper by Procházka and Sejnoha (Applications of Mathematics, 2004). The extension consists of introducing eigenparameters (either eigenstrain or eigenstress) into the formulation. Moreover, these eigenparameters were used in the estimation of bounds with the result that elastic strain is in a certain relation with plastic strains. This appears to be a very restrictive condition (constraint) and a new approach will be presented in this paper based on additional estimates. In the classical approach of Hashin and Shtrikman the overall energies were compared with local energies (on the micro scale level). From this the procedure for evaluation of bounds on overall material properties involving eigenparameters begins. In our case it is possible to consider the eigenparameters as characterizations of plastic behavior of one or more phases in the composite structure. Following this idea, the eigenparameters can describe the current situation in the composite structure and involved in the estimates. In such a way, the eigenparameters can be considered as plastic strains or relaxation stresses, and then the properties of eigenparameters can be taken into consideration. Basically, the former approach of the paper by Hashin-Shtrikman still remains in the body of the presented derivation of the new principles.
\end{abstract}

Keywords: extended Hashin-Shtrikman principles, bounds on overall properties, hereditary problems. 


\section{Introduction}

Eigenstresses and eigenstrains play a very important role in many branches of applied mechanics, e.g., in composites, geotechnics, concrete structures, etc. In previous papers, the authors have formulated an effective approach to the analysis and optimization of inhomogeneous bodies with prescribed boundary displacements or tractions and have used the transformation field analysis for relating the components of stress or strain tensors and of eigenstrains or eigenstresses. The transformation field analysis established by Dvorak and Procházka in [1] has been applied to the localization of stresses and strains in two-phase composites. The eigenstresses stood for relaxation stresses while eigenstrains represented plastic strains. This idea was extended in [2], where applications of a large scale of combinations of internal material situations using the boundary element method with eigenstress of composite structures were considered.

In [3] an interesting attempt at obtaining effective material properties of a nonlinear isotropic composite has been made, using the basic ideas of Hashin and Shtrikman, [4]. A new variational approach was proposed that provides the effective energy potentials of nonlinear composites in terms of the corresponding energy potentials for linear composites with the same microstructural distributions. The first application of the extended $\mathrm{H}-\mathrm{S}$ principles appears in [5], where certain plasticity problems are solved under some assumptions.

Our approach is based on the idea of augmented Hashin-Shtrikman variational principles. This paper deals with the extended primary variational principle for nonhomogeneous bodies. By means of internal parameters, eigenstrains or eigenstresses, involved in $\mathrm{H}-\mathrm{S}$ principles, it is possible to obtain new bounds on mechanical properties of the trial material, increase the bearing capacity of structures, and to minimize the stress excesses.

\section{Basic relations}

We start with basic relations that are valid in mechanics of continuum and are appropriate for our next considerations. A bounded domain is denoted by $\Omega$, $\Gamma \equiv \Gamma_{u} \cup \Gamma_{p}, \Gamma_{u} \cap \Gamma_{p}=0$ being its Lipschitz's boundary, both representing the trial body. On $\Gamma_{u}$ the displacement vector $\boldsymbol{u}=\left\{u_{1}, u_{2}, u_{3}\right\}$ is prescribed, and on $\Gamma_{p}$ the vector of tractions $\boldsymbol{p}=\left\{p_{1}, p_{2}, p_{3}\right\}$ is given. Recall the relation stresses-tractions on the boundary $\Gamma_{p}: p_{i}=\sigma_{i j} n_{j}$, where $\boldsymbol{n} \equiv\left\{n_{1}, n_{2}, n_{3}\right\}$ is the outward unit normal to the boundary and $\sigma_{i j}, \varepsilon_{i j}$ are components of stress and strain tensors, respectively. form

Hooke's law for anisotropic and inhomogeneous fields is introduced in the

$$
\sigma_{i j}(\boldsymbol{x})=L_{i j k l}(\boldsymbol{x}) \varepsilon_{k l}(\boldsymbol{x})+\lambda_{i j}(\boldsymbol{x}), \varepsilon_{i j}(\boldsymbol{x})=M_{i j k l}(\boldsymbol{x}) \sigma_{k l}(\boldsymbol{x})+\mu_{i j}(\boldsymbol{x})
$$


where $\lambda_{i j}$ are components of the eigenstress tensor, $\mu_{i j}$ are the components of the eigenstrain tensor, $\boldsymbol{x}$ is a position at which the material relations are studied, $L_{i j k l}$ are components of the material stiffness tensor and $M_{i j k l}$ describes its compliance material tensor, both with the standard symmetry; the subscripts $i, j, k, l$ run the set $\{1,2,3\}$,

Moreover, we have

$$
L_{i j k l} M_{k l m n}=I_{i j m n}, \quad I_{i j m n}=\frac{1}{2}\left(\delta_{i m} \delta_{j n}+\delta_{i n} \delta_{j m}\right)
$$

where $I_{i j m n}$ characterises the fourth-order unit tensor, $\delta_{i j}$ is the Kronecker delta being equal to one for $i=j$ and zero otherwise.

Note that for a homogeneous and isotropic material the material stiffness matrix has the form

$$
L_{i j k l}=\lambda \delta_{i j} \delta_{k l}+2 \mu I_{i j k l}
$$

where $\lambda$ and $\mu$ are Lame's constants. Instead of $\mu$, the shear modulus $G$ is sometimes introduced.

Comparing both equations (1), we get

$$
\lambda_{i j}=-L_{i j k l} \mu_{k l} \quad, \quad \mu_{i j}=-M_{i j k l} \lambda_{k l}
$$

Kinematic equations may be written as

$$
\varepsilon_{i j}=\frac{1}{2}\left(\frac{\partial u_{i}}{\partial x_{j}}+\frac{\partial u_{j}}{\partial x_{i}}\right)
$$

Note that displacements are said to be kinematically admissible if the relation (5) holds.

Eventually, static equations or equations of equilibrium yield

$$
\frac{\partial \sigma_{i j}}{\partial x_{j}}=0
$$

provided no volume weight forces are taken into account. The last relation has to be taken in the sense of distributions.

Note that one says that the stress tensor is statically admissible or its components are statically admissible, if statistical boundary conditions on $\Gamma_{p}$ are prescribed and (6) is fulfilled.

Substituting the kinematical equations into the equations of equilibrium leads to Lame's equations for the unknown displacement vector $\boldsymbol{u}$, which are written in the sense of distributions:

$$
\frac{\partial}{\partial x_{j}}\left[L_{i j k l}\left(\frac{\partial u_{k}}{\partial x_{l}}+\frac{\partial u_{l}}{\partial x_{k}}-2 \mu_{k l}\right)\right]=0 \quad \text { in } \Omega,
$$

or alternatively 


$$
\frac{\partial}{\partial x_{j}}\left[L_{i j k l}\left(\frac{\partial u_{k}}{\partial x_{l}}+\frac{\partial u_{l}}{\partial x_{k}}-2 \lambda_{k l}\right)\right]=0 \quad \text { in } \Omega
$$

for a given field $\mu_{k l}$, or $\lambda_{k l}$.

Recall that on the part $\Gamma_{u}$ of the boundary $\Gamma$ the displacement vector $\boldsymbol{u}$ is prescribed, and the traction field $\boldsymbol{p}$ is given on $\Gamma_{p}$. Assuming a smooth enough field $\boldsymbol{u} \in \Omega$, we can formulate a variational principle, which is equivalent to the equation (7) or (8):

Lagrange's primary principle: For given tractions $\boldsymbol{p} \in \Gamma_{p}$ find the minimum value of the functional $\Pi_{u}(\boldsymbol{u})=\Pi_{i}(\boldsymbol{u})+\Pi_{e}(\boldsymbol{u})$ on the set of kinematically admissible displacements, where

$$
\begin{gathered}
\Pi_{i}(\boldsymbol{u})=\frac{1}{2} \int_{\Omega} L_{i j k l}(\boldsymbol{x})\left[\varepsilon_{i j}(\boldsymbol{x})-\mu_{i j}(\boldsymbol{x})\right]\left[\varepsilon_{k l}(\boldsymbol{x})-\mu_{k l}(\boldsymbol{x})\right] \mathrm{d} \Omega= \\
\left.=\frac{1}{2} \int_{\Omega} M_{i j k l}(\boldsymbol{x}) \sigma_{i j}(\boldsymbol{x}) \sigma_{k l}(\boldsymbol{x})\right] \mathrm{d} \Omega \\
\Pi_{e}=-\int_{\Gamma_{p}} p_{i}(\boldsymbol{x}) u_{i}(\boldsymbol{x}) \mathrm{d} \Gamma_{p}
\end{gathered}
$$

Here $\Pi_{i}$ is the energy of internal forces, potential energy, whereas $\Pi_{e}$ is the energy of external forces. $\mathrm{W}$ is the density of internal energy.

Assuming the validity of (5), the principle is equivalent to (1), or, if (1) and the boundary condition on $\Gamma_{u}$ are fulfilled, then the variational principle is equivalent to (5).

\section{Extended Hashin-Shtrikman variational principles}

In this section we extend the Hashin-Shtrikman variational principle [4], by introducing both the eigenstrain and eigenstress fields into the formulation. For the sake of simplicity assume that no body forces are present.

The idea of Hashin and Shtrikman consists of introducing new variables $\tau_{i j}$ (components of polarization tensor) to get another free variable that may be used for "the best" estimation of bounds on overall material properties of nonhomogeneous and anisotropic media.

Let us consider again a bounded domain $\Omega$ with its bounded Lipschitz's boundary $\Gamma$ and with subdomains $\Omega_{i}, i=1, \ldots, n$, describing local inhomogeneities.

Following the Hashin and Shtrikman idea, let us split the procedure into two steps. First, let $\varepsilon_{i j}^{0}$ and $\sigma_{i j}^{0}$ be the small strain and the stress fields, respectively. These stresses and strains are related by the linear homogeneous isotropic Hooke's law: 


$$
\sigma_{i j}^{0}=L_{i j k l}^{0} \varepsilon_{k l}^{0}, \quad \text { or } \quad \varepsilon_{i j}^{0}=M_{i j k l}^{0} \sigma_{k l}^{0}
$$

where $L_{i j k l}^{0}$ and $M_{i j k l}^{0}$ are constant components of material stiffnesses and compliances, respectively. Subscripts in (11) run from 1 to 3. It is worth noting that the stresses $\sigma_{i j}^{0}$ are statically admissible, since linear elasticity is considered in the above comparison media of the trial body (the quantities in which are denoted by 0$)$. Similarly, kinematic equations $\left(\varepsilon_{i j}^{0}=1 / 2\left(\frac{\partial u_{i}}{\partial x_{j}}+\frac{\partial u_{j}}{\partial x_{i}}\right)\right)$ are valid to get the proper relation between the components of the strain tensor and the displacement vector. These conditions will be necessary in what follows. In this sense, the quantities with 0 are considered to be given.

In the second step a geometrically identical body is considered, which is anisotropic and inhomogeneous. Displacements $u_{i}$, strains $\varepsilon_{i j}$ and stresses $\sigma_{i j}$ are unknown and the generalized Hooke's law including the eigenstresses $\lambda_{i j}$ is formulated in (1).

Similarly to the classical Hashin-Shtrikman principles, define the symmetric stress polarization tensor $\tau_{i j}$ by

$$
\sigma_{i j}=L_{i j k l}^{0} \varepsilon_{k l}+\tau_{i j}
$$

The definition of polarization tensors follows from a comparison of (12) and (1). Define also

$$
u_{i j}^{\prime}=u_{i j}-u_{i j}^{0}, \quad \varepsilon_{i j}^{\prime}=\varepsilon_{i j}-\varepsilon_{i j}^{0}, \quad \sigma_{i j}^{\prime}=\sigma_{i j}-\sigma_{i j}^{0}
$$

Let us introduce the assumption that the surface displacements are given, so that $u_{i}^{\prime}=0$ on the boundary. Then the variational principle can be formulated.

\section{Generalized Hashin-Shtrikman principles}

Total energy in a composite can be recorded in a primed system, see [4], involving polarization tensor $\tau_{i j}$ and eigenstresses $\lambda_{i j}$ as:

$$
\begin{aligned}
& U\left(\tau_{i j}, \varepsilon_{i j}^{\prime}\right)=U_{0}-\frac{1}{2} \int_{\Omega}\left\{C_{i j k l}\left(\tau_{i j}-\lambda_{i j}\right) *\right. \\
& \left.*\left(\tau_{k l}-\lambda_{k l}\right)-2 \tau_{i j} \varepsilon_{i j}^{0}-\tau_{i j} \varepsilon_{i j}^{\prime}-M_{i j k l} \lambda_{i j} \lambda_{k l}\right\} \mathrm{d} \Omega
\end{aligned}
$$

where

$$
U_{0}=\frac{1}{2} \int_{\Omega} \sigma_{i j}^{0} \varepsilon_{i j}^{0} \mathrm{~d} \Omega=\frac{1}{2} \int_{\Omega} L_{i j k l}^{0} \varepsilon_{i j}^{0} \varepsilon_{k l}^{0} \mathrm{~d} \Omega
$$

and other quantities are denoted as in [4]. It also holds a very important theorem: the functional $U$ attains its absolute maximum if $[\boldsymbol{L}]$ is positive definite, and it attains its absolute minimum if $\boldsymbol{C}+\boldsymbol{M}^{0}$ is negative semidefinite. Applying 
successively unit surface displacements in basic directions on the unit cell we arrive at the equivalent expression to (14):

$$
\begin{aligned}
& U=U_{0}+\frac{9}{2} \frac{A\left(e^{0}\right)^{2}}{1+\alpha_{0} A}+\frac{1}{2} \frac{B\left(e_{i j}^{0}\right)^{2}}{1+\beta_{0} B}+ \\
& +3\left(e^{0}+m\right) l+\left(e_{i j}^{0}+m_{i j}\right) l_{i j}
\end{aligned}
$$

where $A, B, \alpha_{0}$, and $\beta_{0}$ are material constants depending on fiber ratio, $l$ and $m$ are isotropic and $l_{i j}$ and $m_{i j}$ are deviatoric parts of eigenstress and eigenstrain tensors, $e^{0}$ and $e_{i j}^{0}$ are the similar quantities for strains.

\section{Example: time dependent problem}

Introducing time dependent law we start with the extended Hooke's law, which is in this case dependent on time:

$$
\sigma_{r s}(t)=L\left(\varepsilon_{r s}(t)\right)+\lambda_{r s}(t)=\lambda \varepsilon_{k k}(t) \delta_{r s}+2 G \varepsilon_{r s}(t)+\lambda_{r s}(t)
$$

From (16) the inverse relation leads us to:

$$
\begin{aligned}
\varepsilon_{r s}(t) & =\frac{\sigma_{r s}(t)-\lambda_{r s}(t)}{2 G}-\frac{\sum_{k}\left(\sigma_{k k}(t)-\lambda_{k k}(t)\right) \delta_{r s}}{3 K}= \\
& =\frac{\sigma_{r s}(t)}{2 G}-\frac{\sum_{k}\left(\sigma_{k k}(t)\right) \delta_{r s}}{3 K}-\frac{\lambda_{r s}(t)}{2 G}+\frac{\sum_{k}\left(\lambda_{k k}(t)\right) \delta_{r s}}{3 K}= \\
& =M\left(\sigma_{r s}(t)\right)-M\left(\lambda_{r s}(t)\right)=M\left(\sigma_{r s}(t)\right)+\mu_{r s}(t),
\end{aligned}
$$

where

$$
M\left(\sigma_{r s}(t)\right)=\frac{\sigma_{r s}(t)}{2 G}-\frac{\sum_{k}\left(\sigma_{k k}(t)\right) \delta_{r s}}{3 K}, \quad \mu_{r s}(t)=-M\left(\lambda_{r s}(t)\right)
$$

The plasticity path is defined in dependence on time as; see [6] and [7]:

$$
\boldsymbol{\mu}(t)=\boldsymbol{\varepsilon}^{p}(t)+\boldsymbol{\varepsilon}^{v p}(t),
$$

where $\boldsymbol{\varepsilon}^{p}(t)=A(\boldsymbol{\sigma}(t))^{n} t$ is the plastic term and $\boldsymbol{\varepsilon}^{v p}(t)=\left[\frac{\boldsymbol{H}(t)}{B}\right]^{1 / q}$ is the viscoplastic term, $\boldsymbol{H}(t)$ is the overloading in time $t$ and $A, n, B, q$ are material constants.

Hooke's law in (17) and (18) can be written in dependence on time as:

$$
\boldsymbol{\varepsilon}(t)=\boldsymbol{M}(\boldsymbol{\sigma}(t))+A(\boldsymbol{\sigma}(t))^{n} t+\left[\frac{\boldsymbol{H}(t)}{B}\right]^{1 / q}
$$


The time interval is divided into $N$ subintervals, for which values of $\sigma(i)$, $\varepsilon(i)$ and $\boldsymbol{H}(i)$ are prescribed. It appears that it is possible to define $\boldsymbol{H}(i)$ as the difference in the dynamical deviation of stresses from the stresses due to assumed constant load $\left(\sigma^{d}(i)-\sigma^{s}(i)\right)$.

From (21) relations for the calculation of coefficients $a^{p}(i), b^{p}(i)$ in step $i$ are derived:

$$
a^{p}(i)=\frac{1}{e^{0}}\left[A_{a}\left(s^{i}\right)^{n} \frac{i}{i^{\max }}+\left[\frac{h^{i}}{B_{a}}\right]^{1 / q}\right], \quad b^{p}(i)=\frac{1}{e_{r s}^{m^{0}}}\left[A_{b}\left(s_{r s}^{m^{i}}\right)^{n} \frac{i}{i^{\max }}+\left[\frac{h_{r s}^{m^{i}}}{B_{b}}\right]^{1 / q}\right],
$$

where $s^{i}, s_{r s}^{m^{i}}$ are components of stresses and $h, h_{r s}^{d}$ are components of overloading, both in step $i$, and moreover $A_{a}, A_{b}, n, B_{a}, B_{b}, q$ are material characteristics, $i$ denotes the step (discretization of time $t$ ).

If $s^{i}, s_{r s}^{m^{i}}$ are constant, then (22) turns to:

$$
a^{p}(i)=3 A_{a} K_{1}\left(s^{i}\right)^{n-1} \frac{i}{i^{\max }}, \quad b^{p}(i)=2 A_{b} G_{1}\left(s_{r s}^{m^{i}}\right)^{n-1} \frac{i}{i^{\max }},
$$

Remark: the exponent $n$ and multiplier $A$ are known from experiments.

If $n=1, q=1$, then (22) can be rewritten as:

$$
\begin{gathered}
a^{p}(i)=3 A_{a} K_{1} \frac{i}{i^{\max }}+K_{1} \frac{h^{i}}{B_{a}}, \quad b^{p}(i)=2 A_{b} G_{1} \frac{i}{i^{\max }}+G_{1} \frac{h_{r s}^{m^{i}}}{B_{b}}, \\
h^{i}=\frac{e^{0}-e^{\Delta}(i)}{e^{0}}, \quad h_{r s}^{m^{i}}=\frac{e_{r s}^{m^{0}}-e_{r s}^{m^{4}}(i)}{e_{r s}^{m^{0}}}
\end{gathered}
$$

The estimates of bounds of $K^{* i}$ and $G^{* i}$ in step $i$ are given as:

$$
\begin{aligned}
& K_{1}^{* i} \leq K^{* i} \leq K_{2}^{* i}, \\
& K_{1}^{* i}=K_{1}+\frac{A_{1}}{1+\alpha_{1} A_{1}}-A^{p^{i}}, \quad K_{2}^{* i}=K_{n}+\frac{A_{n}}{1+\alpha_{n} A_{n}}-A^{p^{i}}, \\
& A^{p^{i}}=K_{p} a^{p}(i) v_{p}\left(2-a^{p}(i) v_{p}\right), \quad \alpha_{1}=-\frac{3}{3 K_{1}+4 G_{1}}, \\
& \alpha_{n}=-\frac{3}{3 K_{n}+4 G_{n}},
\end{aligned}
$$

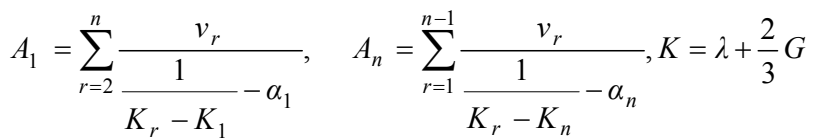




$$
\begin{aligned}
& G_{1}^{* i} \leq G^{* i} \leq G_{2}^{* i}, \\
& G_{1}^{* i}=G_{1}+\frac{1}{2} \frac{B_{1}}{1+\beta_{1} B_{1}}-B^{p^{i}}, \quad G_{2}^{* i}=G_{n}+\frac{1}{2} \frac{B_{n}}{1+\beta_{n} B_{n}}-B^{p^{i}}, \\
& B^{p^{i}}=G_{p} b^{p}(i) v_{p}\left(2-b^{p}(i) v_{p}\right), \quad \beta_{1}=-\frac{3\left(K_{1}+2 G_{1}\right)}{5 G_{1}\left(3 K_{1}+4 G_{1}\right)}, \\
& \beta_{n}=-\frac{3\left(K_{n}+2 G_{n}\right)}{5 G_{n}\left(3 K_{n}+4 G_{n}\right)}, \\
& B_{1}=\sum_{r=2}^{n} \frac{v_{r}}{2\left(G_{r}-G_{1}\right)}-\beta_{1}, \quad B_{n}=\sum_{r=1}^{n-1} \frac{v_{r}}{2\left(G_{r}-G_{n}\right)}-\beta_{n}
\end{aligned}
$$

In Fig. 1 the application to two-phase material is illustrated.

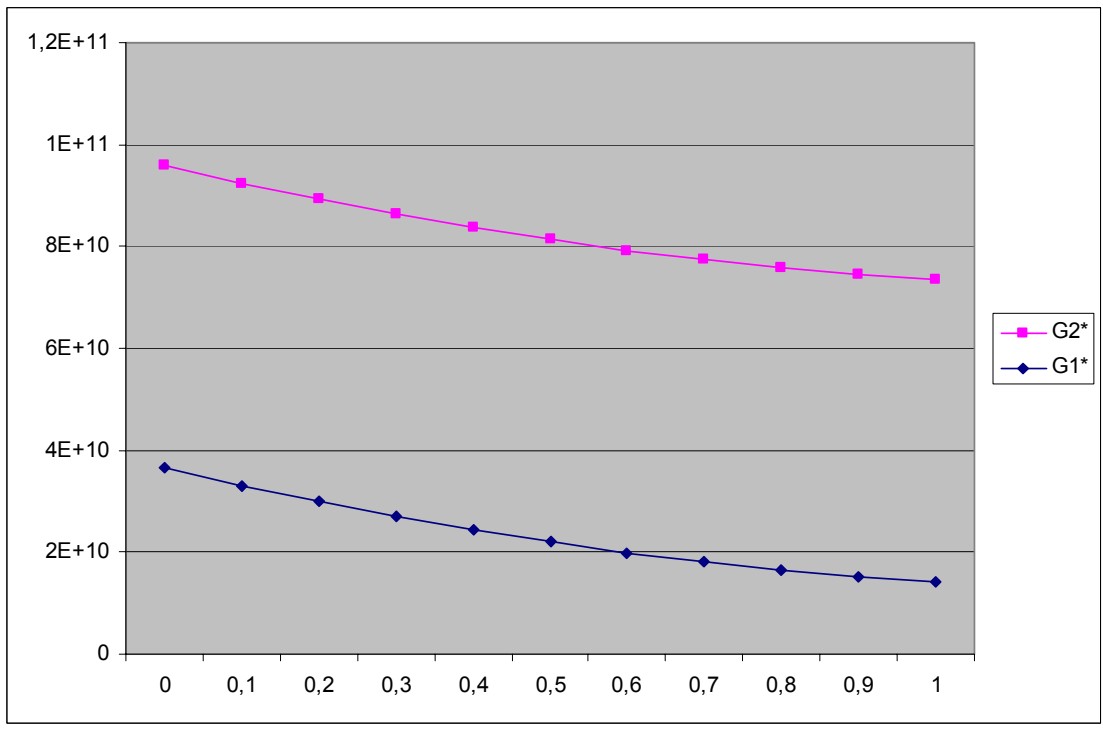

Figure 1: Dependence of bounds $G_{2}^{* i}$ a $G_{1}^{* i}$ on time $t$ for $A_{b}=2 * 10^{-11}$ and $n=1$, and for $v_{\text {matrix }}=0.8, v_{\text {phase }}=0.2$.

\section{Conclusions}

In the paper generalized $\mathrm{H}-\mathrm{S}$ bounds on overall modules of a two phase composite were derived. In the plastic region, the lower bound on the shear modules is basically lowered under some conditions. The most common case is considered; one phase behaves as brittle, while the other is plastic. A large class of isotropic plastic rules can be involved in the study presented here. It is 
obviously possible to derive dependence of intrinsic parameters of a concrete plastic rule and the multiplier of plastic strains with respect to the elastic ones. For example, Mises with hardening can be studied with the approach provided in this paper. Time dependency affecting material properties within a time period is taken into account and added to the model.

\section{Acknowledgements}

The financial support of GACR, project No. 103/07/0304, is appreciated. The support of CIDEAS at CTU Prague is also acknowledged.

\section{References}

[1] Dvorak, G.J., Procházka, P.P. Thin-walled composite cylinders with optimal fiber prestress. Composites Part B, 27B, 1996, 643-649

[2] Prochazka, P.P., Sejnoha, J. Behavior of composites on bounded domain. BE Communications 7, 1996, 6-8

[3] Prochazka, P.P., Sejnoha, J. Extended Hashin-Shtrikman variational principles. Applications of Mathematics, No. 4, 49, 2004, 357-372.

[4] Hashin, Z., Shtrikman, S. A variational approach to the theory of the elastic behaviour of polycristals. J. Mech. Phys. Solids 10, 1962, 343-352

[5] Toman, M. Thesis for PhD, CTU Prague, 2007 (in Czech)

[6] Katsuhisa Ohta: Time-dependent variational principle with constraints, Chemical Physics Letters, Volume 329, Issues 3-4, 20 October 2000, Pages 248-254.

[7] M. Al-Haika, M.R. Vaghara, H. Garmestania, M. Shahawy: Viscoplastic analysis of structural polymer composites using stress relaxation and creep data, Department of Mechanical Engineering, FAMU-FSU College of Engineering, Center for Materials Research and Technology, 10, 2000. 